\title{
On Moments of the Power Series Distributions
}

\author{
Slavko Simic*
}

Mathematical Institute SANU, Kneza Mihaila 36, 11000 Belgrade, Serbia

\begin{abstract}
For the Power Series Distributions generated by an arbitrary entire function of finite order, applying methods of Karamata's Theory of Regular Variation, we obtain asymptotic behavior of its moments. As an illustration, we calculate the moments of distributions generated by the class of Mittag-Leffler functions of which the well-known Poisson Law is just a special case.
\end{abstract}

Keywords: Distribution moments, entire functions of finite order, regular variation, karamata.

\section{INTRODUCTION}

1.1. The classical discrete Poisson Law with parameter $\lambda$ $>0$ is given by

$$
P_{\lambda}(X=n)=\frac{\lambda^{n} e^{-\lambda}}{n !}, n=0,1,2, \ldots
$$

Asymptotic behavior of its moments is well-known

$$
E_{\lambda} X^{k}=\sum_{n} n^{k} P_{\lambda}(X=n) \sim \lambda^{k}=\left(E_{\lambda} X\right)^{k}(\lambda \rightarrow \infty) .
$$

In this article we shall give a generalization of the above assertion and prove that the same asymptotic relation is valid for any discrete distribution generated by an entire function of finite order.

Namely, denote by $A_{\rho}$ the class of transcendental entire functions with positive Taylor coefficients and of finite order $\rho, 0 \leq \rho<\infty$.

(Note that an entire function is transcendental if it is not a polynomial i. e. if it has infinitely many non-zero Taylor coefficients).

Definition 1.1. For arbitrary $f(z)=\sum a_{n} z^{n}, f \in A_{\rho}$, define the law $F_{f}$ with parameter $\lambda>0$ by

$$
P_{\lambda}(X=n)=a_{n} \lambda^{n} / f(\lambda), n=0,1,2, \ldots
$$

Then the law $F_{f}$ is a Power Series Distribution generated by $f \in A_{\rho}(\operatorname{cf}[1])$.

Our aim here is to obtain asymptotic behavior of its $k$-th moment $E_{\lambda} X^{k}(\lambda \rightarrow \infty)$, where

$$
E_{\lambda} X^{k}=\sum n^{k} P_{\lambda}(X=n)=\sum n^{k} a_{n} \lambda^{n} / f(\lambda), k=1,2, \ldots
$$

Evidently,

$$
E_{\lambda} X=\sum n a_{n} \lambda^{n} / f(\lambda)=\lambda f^{\prime}(\lambda) / f(\lambda) .(0.1)
$$

In general, consider the sequence of functions $f_{k}(\lambda)$ defined recursively by

*Address correspondence to this author at the Mathematical Institute SANU, Kneza Mihaila 36, 11000 Belgrade, Serbia; E-mail: ssimic@turing.mi.sanu.ac.yu

2000 Mathematics Subject Classification. 60E05, 30D15.

$$
f_{k}(\lambda)=\lambda f_{k-1}^{\prime}(\lambda), k=1,2, \ldots ; f_{0}(\lambda)=f(\lambda) .
$$

Then $f_{k}(\lambda)=\sum n^{k} a_{n} \lambda^{n}$ and

$$
\mathrm{E}_{\lambda} X^{k}=f_{k}(\lambda) / f(\lambda), k=1,2, \cdots(0.2)
$$

1.2. We shall use in the sequel the concept of Karamata's regularly varying functions.

Definition 1.2. A positive measurable function $\ell(\cdot)$ is slowly varying if the asymptotic equivalence

$$
\ell(t x) \sim \ell(x)(x \rightarrow \infty),
$$

holds for each $t>0$.

Some examples of slowly varying functions are $\log ^{a} x$; $\log ^{b}(\log x) ; \exp \left(\log ^{c} x\right) ; \exp (\log x / \log \log x), a, b \in \mathbf{R}, 0<c$ $<1$.

Functions $g(\cdot)$ of the form $g(x)=x^{\mu} \ell(x)$ are regularly varying $\left(g \in R_{\mu}\right)$ with index $\mu \in \mathbf{R}(\operatorname{cf}[2]$, p. 18).

From those definitions follows

Proposition 1.1. A positive measurable function $g(\cdot)$ belongs to the class $R_{\mu}$ if the asymptotic relation

$$
\frac{g(t x)}{g(x)} \rightarrow t^{\mu} \quad(x \rightarrow \infty)
$$

holds for each $t>0$.

Moreover, the relation (1.1) holds uniformly on each compact t-set in $(0, \infty)(\mathrm{cf}[2]$, p. 6$)$.

Theory of Regular Variation is very well-developed and has many applications in Analysis, Probability Theory, Number Theory etc (cf [2, 3]).

The so-called Theorem on approximation by a regularly varying function will play an important role in the sequel (cf [2], p. 81; [4]).

Proposition 1.2. Let $h$ be a positive function with the property

$$
\limsup _{x \rightarrow \infty} \frac{\log h(x)}{\log x}=\rho, 0 \leq \rho<\infty ;
$$

then there exists $g \in R_{\rho}$ such that $g(x) \geq h(x)$ and 


$$
\limsup _{x \rightarrow \infty} g(x) / h(x)=1
$$

1.3. Now we give some remarks from the Theory of Entire Functions. As is well-known (cf $[2,4,5])$, the order $\rho$ of an entire function $f$ is defined by

$$
\rho=\limsup _{\lambda \rightarrow \infty} \frac{\log \log M_{f}(\lambda)}{\log \lambda},
$$

where $M_{f}(\lambda)=\max _{|z|=\lambda}|f(z)|$ is the maximum moduli of $f$ on the circle $|z|=\lambda$.

But, if $f \in A_{\rho}$, we have

$$
|f(z)|_{|z|=\lambda}=\left|f\left(\lambda e^{i \phi}\right)\right|=\left|\sum a_{n} \lambda^{n} e^{i n \phi}\right| \leq \sum a_{n}\left|\lambda^{n} e^{i n \phi}\right|=f(\lambda) .
$$

Therefore, in this case $M_{f}(\lambda)=f(\lambda)$ and we get

Definition 1.3. If $f \in A_{\rho}$, then

$$
\rho=\limsup _{\lambda \rightarrow \infty} \frac{\log \log f(\lambda)}{\log \lambda} \text { (3.1). }
$$

This definition coincides with another one given in Lemma 3.2. below, as is shown in $([6,7], \mathrm{p} .17)$.

\section{RESULTS}

We shall prove the following assertions on asymptotic behavior of the moments of the law $F_{f}$.

Theorem 2.1. For each $n \in \mathbb{N}$,

$$
E_{\lambda} X^{n+1} / E_{\lambda} X^{n} \sim E_{\lambda} X(\lambda \rightarrow \infty) .
$$

Theorem 2.2. For each $\alpha>0$ we have

$$
E_{\lambda} X^{\alpha} \sim\left(E_{\lambda} X\right)^{\alpha}=\left(\lambda f^{\prime}(\lambda) / f(\lambda)\right)^{\alpha}(\lambda \rightarrow \infty) .
$$

It is said that an entire function $g$ of finite order $\rho$ is of complete regular growth if $\log g \in R_{\rho}$ i. e. $\log g(r) \sim r^{\rho} \ell(r)$ $(r \rightarrow \infty)$ for some slowly varying function $\ell$.

Since standard examples of $f \in A_{\rho}$ are of complete regular growth, we shall give a more precise statement of Theorem 2.2 in this case. The link is given by the next

Theorem 2.3. For $f \in A_{\rho}, \rho>0$, the following are equivalent

(i) $\log f(\lambda) \sim \lambda^{\rho} \ell(\lambda)$; (ii) $\mathrm{E}_{\lambda} \mathrm{X} \sim \rho \lambda^{\rho} \ell(\lambda)(\lambda \rightarrow \infty)$.

Combining the last two assertions we obtain

Theorem 2.4. If $f \in A_{\rho}$ is of complete regular growth, then for $\alpha>0$,

$$
E_{\lambda} X^{\alpha} \sim \rho^{\alpha}(\log f(\lambda))^{\alpha} \sim \rho^{\alpha} \lambda^{\alpha \rho}(\ell(\lambda))^{\alpha}(\lambda \rightarrow \infty) .
$$

\section{PROOFS} $=1$.

The crucial part in the proof of Theorem 2.1. is the case $n$

Lemma 3.1. $E_{\lambda} X$ and $E_{\lambda} X^{2} / E \lambda X$ are monotone increasing in $\lambda$ and unbounded.

Proof. Since

$$
\lambda \frac{d}{d \lambda}\left(E_{\lambda} X\right)=E_{\lambda} X^{2}-\left(E_{\lambda} X\right)^{2}>0
$$

we conclude that $E_{\lambda} X$ is monotone increasing and unbounded; otherwise, there exists $a>0$ such that $E_{\lambda} X<a$ for $\lambda>0$. By $(0.1)$, we get $f^{\prime}(\lambda) / f(\lambda)<a / \lambda$ and integrating, we find $f(\lambda)=O\left(\lambda^{a}\right)$. Hence in this case $f$ is a polynomial, not a transcendental function.

The proof of the second assertion goes analogously.

Since $E_{\lambda} X^{2}>\left(E_{\lambda} X\right)^{2}$, it follows that

$\liminf _{x \rightarrow \infty} \frac{E_{\lambda} X^{2}}{\left(E_{\lambda} X\right)^{2}} \geq 1$

To prove that $\lim \sup _{\lambda \rightarrow \infty} E_{\lambda} X^{2} /\left(E_{\lambda} X\right)^{2} \leq 1$, denote

$E_{\lambda} X=f_{1}(\lambda) / f_{0}(\lambda)=h_{0}(\lambda) ; E_{\lambda} X^{2} / E_{\lambda} X=f_{2}(\lambda) / f_{1}(\lambda)=h_{1}(\lambda)$.

Then

$E_{\lambda} X^{2} /\left(E_{\lambda} X\right)^{2}=h_{1}(\lambda) / h_{0}(\lambda)$ and $h_{i} \uparrow^{\infty}, i=0,1$.

We also need the following classical statement (cf [6], Vol. 2, p. 17).

Lemma 3.2. If $f \in A_{\rho}$, then

$$
\limsup _{x \rightarrow \infty} \frac{\log h_{0}(\lambda)}{\log \lambda}=\rho
$$

Combining this and Proposition 1.2, we find out that there exists a regularly varying function $g \in R_{\rho}$ such that

$$
h_{0}(\lambda) \leq g(\lambda) ; \limsup _{x \rightarrow \infty} \frac{g(\lambda)}{h_{0}(\lambda)}=1 .
$$

Therefore, for arbitrary $C>1$ we get

$$
g(\lambda) / C<h_{0}(\lambda) \leq g(\lambda)\left(\lambda>\lambda_{0}(C)\right),
$$

and consequently, for each $t>1$,

$$
g(\lambda t) / C<h_{0}(\lambda t) \leq g(\lambda t)\left(\lambda>\lambda_{0}(C)\right) .
$$

Hence

$$
\frac{h_{0}(\lambda t)}{h_{0}(\lambda)}<C \frac{g(\lambda t)}{g(\lambda)}\left(\lambda>\lambda_{0}(C)\right),
$$

i. e., by Proposition 1.1, we finally obtain that

$$
\frac{h_{0}(\lambda t)}{h_{0}(\lambda)}<C^{2} t^{\rho}\left(\lambda>\lambda_{1}(C)\right),
$$

holds for each $t>1$.

Another assertion is of importance.

Lemma 3.3. For each $t>1, \lambda>0$, we have

$$
h_{i}(\lambda) \log t<\log \frac{f_{i}(\lambda t)}{f_{i}(\lambda)}<h_{i}(\lambda t) \log t, i=0,1 .
$$

Proof. By Lemma 3.1, $h_{i}$ is monotone increasing; thus $\log \frac{f_{i}(\lambda t)}{f_{i}(\lambda)}=\int_{\lambda}^{\lambda t} h_{i}(u) d u / u>h_{i}(\lambda) \int_{\lambda}^{\lambda t} d u / u=h_{i}(\lambda) \log t$, and analogously, $\log \frac{f_{i}(\lambda t)}{f_{i}(\lambda)}<h_{i}(\lambda \mathrm{t}) \log t, i=0,1$. 
Now, since $h_{0}=f_{1} / f_{0}$, combining Lemma 3.3 and (4), for each $t>1$ we obtain

$h_{1}(\lambda) \log t<\log \frac{f_{1}(\lambda t)}{f_{1}(\lambda)}<\log \left(C^{2} t^{\rho}\right)+\log \frac{f_{0}(\lambda t)}{f_{0}(\lambda)}<\log \left(C^{2} t^{\rho}\right)$

$$
+h_{0}(\lambda \mathrm{t}) \log t
$$

$<\log \left(C^{2} t^{\rho}\right)+\left(C^{2} t^{\rho} \log t\right) h_{0}(\lambda), \lambda>\lambda_{1}(C)$.

Since $h_{0}(\lambda) \rightarrow \infty$ as $\lambda \rightarrow \infty$, choosing in (5) $t=C$ if $\rho=0$ and $t=\mathrm{C}^{1 / \rho}$ for $\rho>0$, we get

$$
h_{1}(\lambda) / h_{0}(\lambda)<C^{3}+o(1)(\lambda \rightarrow \infty) .
$$

Because $C>1$ is arbitrary, by (3) we conclude that

$$
\limsup _{\lambda \rightarrow \infty} \frac{E_{\lambda} X^{2}}{\left(E_{\lambda} X\right)^{2}} \leq 1
$$

and the proof of Theorem 2.1 in the case $n=1$ is done.

To prove this theorem in general, note that $f \in A_{\rho}$ implies $f^{(\mathrm{n})} \in A_{\rho}, n=1,2, \ldots$.

Indeed, that all derivatives of an entire function of order $\rho$ are also of order $\rho$ is a classical result and can be found in [5], p. 265. Secondly, since all Taylor coefficients of $f$ are positive then, evidently, all Taylor coefficients of $f^{(n)}$ are also positive. Therefore we conclude that $f \in A_{\rho}$ implies $f^{(n)} \in A_{\rho}$.

Also

$$
\frac{f(\lambda) f^{\prime \prime}(\lambda)}{\left(f^{\prime}(\lambda)\right)^{2}}=\frac{E_{\lambda} X^{2}}{\left(E_{\lambda} X\right)^{2}}-\frac{1}{E_{\lambda} X} \rightarrow 1(\lambda \rightarrow \infty),
$$

because $E_{\lambda} X \rightarrow \infty$ as $\lambda \rightarrow \infty$.

Since (6) holds for each $f \in A_{\rho}$, replacing $f$ by $f^{(n-1)}$, we get

$$
\frac{f^{(n+1)}(\lambda)}{f^{(n)}(\lambda)} \sim \frac{f^{(n)}(\lambda)}{f^{(n-1)}(\lambda)}(\lambda \rightarrow \infty)
$$

But $f_{n}(\lambda) \sim \lambda^{n} f^{(n)}(\lambda)(\lambda \rightarrow \infty)(\operatorname{cf}[3]$, Vol. I, p. 36). Hence by (0.2) and (7) as $\lambda \rightarrow \infty$,

$$
\begin{aligned}
& \frac{E_{\lambda} X^{n+1}}{E_{\lambda} X^{n}}=\frac{f_{n+1}(\lambda)}{f_{n}(\lambda)} \sim \frac{\lambda^{n+1} f^{(n+1)}(\lambda)}{\lambda^{n} f^{(n)}(\lambda)} \sim \frac{\lambda^{n} f^{(n)}(\lambda)}{\lambda^{n-1} f^{(n-1)}(\lambda)} \sim \\
& \frac{f_{n}(\lambda)}{f_{n-1}(\lambda)}=\frac{E_{\lambda} X^{n}}{E_{\lambda} X^{n-1}}
\end{aligned}
$$

Therefore we get

$$
\frac{E_{\lambda} X^{n+1}}{E_{\lambda} X^{n}} \sim \frac{E_{\lambda} X^{n}}{E_{\lambda} X^{n-1}} \sim \cdots \sim \frac{E_{\lambda} X^{2}}{E_{\lambda} X} \sim E_{\lambda} X .
$$

Proof of Theorem 2.2. A simple consequence of the previous theorem is

Lemma 3.4. For each $n \in \mathbb{N}$, we have

$$
E_{\lambda} X^{n} \sim\left(E_{\lambda} X\right)^{n}(\lambda \rightarrow \infty) .
$$

Proof. Indeed,

$$
E_{\lambda} X^{\mathrm{n}} / E_{\lambda} X=\prod_{k=1}^{n-1}\left(E_{\lambda} X^{k+1} / E_{\lambda} X^{k}\right) \sim\left(E_{\lambda} X\right)^{n-1},
$$

i. e. $E_{\lambda} X^{n} \sim\left(E_{\lambda} X\right)^{n}(\lambda \rightarrow \infty)$.

For the rest of the proof we shall apply well-known Lyapunov moments inequality.

Lemma 3.5. For $r>s>t>0$, we have

$$
\left(E X^{s}\right)^{r-t} \leq\left(E X^{t}\right)^{r-s}\left(E X^{r}\right)^{s-t} .
$$

Let $n>\alpha>n-1, n \in \mathbf{N}$. Then the Lyapunov's inequality and Lemma 3.4 give

$$
\begin{gathered}
E_{\lambda} X^{\alpha} \leq\left(E_{\lambda} X^{n-1}\right)^{n-\alpha}\left(E_{\lambda} X n\right)^{\alpha-n+1} \sim\left(E_{\lambda} X\right)^{(n-1)(n-\alpha)}\left(E_{\lambda} X\right)^{n(\alpha-n+1)}= \\
\left(E_{\lambda} X\right)^{\alpha} .
\end{gathered}
$$

Hence

$$
\limsup _{\lambda \rightarrow \infty} \frac{E_{\lambda} X^{\alpha}}{\left(E_{\lambda} X\right)^{\alpha}} \leq 1 \text {. }
$$

Now, let $r=n+1, s=n, t=\alpha$. We get

$$
\left(E_{\lambda} X^{n}\right)^{n+1-\alpha} \leq\left(E_{\lambda} X^{\alpha}\right)\left(E_{\lambda} X^{n+1}\right)^{n-\alpha}
$$

i. e.,

$$
\begin{gathered}
E_{\lambda} X^{\alpha} \geq\left(\mathrm{E}_{\lambda} X^{n}\right)^{n+1-\alpha}\left(E_{\lambda} X^{n+1}\right)^{\alpha-n} \sim\left(E_{\lambda} X\right)^{n(n+1-\alpha)}\left(E_{\lambda} X\right)^{(n+1)(\alpha-n)}= \\
\left(E_{\lambda} X\right)^{\alpha} .
\end{gathered}
$$

Therefore

$$
\liminf _{\lambda \rightarrow \infty} \frac{E_{\lambda} X^{\alpha}}{\left(E_{\lambda} X\right)^{\alpha}} \geq 1
$$

and the proof of Theorem 2.2 is done.

\section{Proof of Theorem 2.3}

(i) $\Rightarrow$ (ii): By Lemma 1.1 and (0.1), for $x>\lambda>y>0$, we get

$$
\log f(x)-\log f(y)=\int_{y}^{x}\left(E_{\lambda} X\right) d \lambda / \lambda\left\{\begin{array}{l}
\leq E_{x} X \log (x / y) ; \\
\geq E_{y} X \log (x / y)
\end{array}\right.
$$

Putting in (8) $x=t y, \mathrm{t}>1$ and $y=t x, 0<t<1$, we obtain

$E_{x} X\left\{\begin{array}{l}\leq \frac{\log f(t x)-\log f(x)}{\log t}, t>1 ; \\ \geq \frac{\log f(x)-\log f(t x)}{\log (1 / t)}, 0<t<1 .\end{array}\right.$

Therefore, by Definition 1.2,

$\limsup _{x \rightarrow \infty} \frac{E_{x} X}{x^{\rho} \ell(x)} \leq \frac{1}{\log t}\left(\lim _{x \rightarrow \infty} \frac{\log f(t x)}{x^{\rho} \ell(x)}-\lim _{x \rightarrow \infty} \frac{\log f(x)}{x^{\rho} \ell(x)}\right)=$

$\frac{t^{\rho}-1}{\log t}$

and analogously,

$\liminf _{x \rightarrow \infty} \frac{E_{x} X}{x^{\rho} \ell(x)} \geq \frac{1-t^{\rho}}{\log (1 / t)}, 0<t<1$.

Now, putting $t \downarrow 1$ in (9) and $t \uparrow 1$ in (10), the proof of the above assertion follows.

(ii) $\Rightarrow$ (i): Since $E_{s} X=\rho s^{\rho} \ell(s) \pi(s)$, where $\pi(s) \rightarrow 1$ as $s \rightarrow \infty$, we conclude that $m(s):=\ell(s) \pi(s)$ is slowly varying. As $E_{S} X$ is monotone increasing on $s \geq 1$, we have $E_{s} X \leq E_{T} X$ on $[1, \mathrm{~T}]$. So, 


$$
m(s)=\frac{E_{s} X}{\rho s^{\rho}}<\frac{E_{T} X}{\rho}, s \in[1, T]
$$

i.e. $m(s)$ is locally bounded on $[1, \infty)$. [2]),

Hence by Karamata's Theorem (Prop.1.5.8 on p.26 of

$$
\log f(\lambda)=\int_{1}^{\lambda}\left(E_{s} X\right) d s / s+O(1)=\int_{1}^{\lambda} \rho s^{\rho-1} m(s) d s+O(1) \sim \lambda^{\rho} m(\lambda),
$$

i.e.

$$
\log f(\lambda) \sim \lambda^{\rho} \ell(\lambda)(\lambda \rightarrow \infty)
$$

as required.

Finally, the assertion from Theorem 2.4 is a consequence of Theorems 2.2 and 2.3.

As an illustration, we evaluate the asymptotic moments behavior of the Power Series Distributions generated by Mittag-Leffler functions $M_{t}(z)$,

$$
M_{t}(z):=\sum z^{n} / \Gamma(1+n t), t>0 .
$$

Theorem 3.1. For a class of Power Series Distributions generated by Mittag-Leffler functions $M_{t}(z)$, we have that

$$
E_{\lambda} X^{\alpha}:=\frac{1}{M_{t}(\lambda)} \sum \frac{n^{\alpha}}{\Gamma(1+n t)} \lambda^{n} \sim \frac{\lambda^{\alpha / t}}{t^{\alpha}} ; \alpha, t>0(\lambda \rightarrow \infty) .
$$

Proof. It is well-known (cf [2], p. 329) that

$$
M_{t}(\lambda) \sim \exp \left(\lambda^{1 / t}\right) / t(\lambda \rightarrow \infty) .
$$

By (3.1) we find out that $M_{t}(\lambda)$ is of order $\rho=1 / t$ and, since all Taylors coefficients are positive, we conclude that $M_{t}(\lambda) \in A_{1 / t}$.

Also, since $\log M_{t}(\lambda) \sim \lambda^{1 / t}(\lambda \rightarrow \infty)$, applying the assertion of Theorem 2.4 with $\ell(t)=1$, the above result follows.

\section{CONCLUSION}

Question of moments convergence is a difficult one and entirely depends on characteristics of the concrete probability law. But if $E X_{n} \rightarrow \infty(n \rightarrow \infty)$ then, due to Jensen's inequality, all other moments are also unbounded and there arise the problem of their asymptotic evaluation. In this article we establish asymptotic behavior of the moments of probability laws generated by an entire function of finite order. Namely, we proved that for each $\alpha, \alpha \in \mathbb{R}^{+}$, the asymptotic relation $E X_{\lambda}^{\alpha} \sim\left(E X_{\lambda}\right)^{\alpha}$ holds as $\lambda \rightarrow \infty$. This formula should be of valuable interest for researchers in the field particularly because, up to our knowledge, there are no similar results in literature. Further investigation of the quantity $w_{\alpha}(\lambda):=\frac{E X_{\lambda}^{\alpha}}{\left(E X_{\lambda}\right)^{\alpha}}-1$ seems to be interesting. For instance, by a result from [8] it follows that $\frac{w_{\alpha}(\lambda)}{\alpha(\alpha-1)}$ is logarithmically convex in $\alpha$.

\section{REFERENCES}

[1] N.L. Johnson and S. Kotz, A.W. Kemp, Univariate Discrete Distributions. John Wiley and Sons, 1992.

[2] N.H. Bingham, C.M. Goldie and J.I. Teugels, Regular Variation. Cambridge Univ Press, 1989.

[3] E. Seneta, Regularly Varying Functions. Springer-Verlag, NY, 1976 .

[4] B.J. Levin, "Distribution of zeros of entire functions", American Mathematical Society Providence, 1964.

[5] E.C. Titchmarsh, The Theory of Functions. Oxford University Press, 1939.

[6] G. Polya and G. Szego, Aufgaben und Lehrsatze aus der Analysis. Springer-Verlag, NY, vol. 1, no. 2, 1964.

[7] G. Polya and G. Szego, Problems and Theorems in Analysis I, (Series. Integral Calculus. Theory of Functions). Springer-Verlag, NY, 1964.

[8] S. Simic, "On a new moments inequality”, Stat. Probab. Lett., vol. 78, pp. 2671-2678, 2008. 